\title{
O conceito de constante de equilíbrio químico sua introdução
}

O conceito de equilíbrio químico é um dos mais importantes a introduzir a nível do ensino secundário. Não podendo recorrer, no entanto, às adequadas bases teóricas da termodinâmica é feito em geral, utilizando aproximações que conduzem por vezes a falsos conceitos, o que origina dificuldades e confusões aos estudantes.

A forma tradicional de apresentar o equilibrio químico pelo estabelecimento da constante de equilíbrio com base em considerações cinéticas (que não distinguem claramente estequeometria de mecanismo de reacção) ainda aparece em muitos textos [1], embora a discussão do seu abandono já seja bastante antiga [2].

Uma das incorrecções mais frequentes que deriva desta aproximação é a atribuição de unidades à constante de equilíbrio. Pretende-se com esta comunicação clarificar o conceito de constante de equilíbrio, bem como a sua introdução.

Um equilíbrio é definido pela igualdade de temperaturas, pressões e potenciais químicos das espécies químicas envolvidas. Estas condições conduzem ao mínimo da energia de Gibbs para o sistema em causa. Para uma dada reacção química, a pressão e temperatura constantes, do tipo

$$
\nu_{1} \mathbf{A}_{1}+\nu_{2} \mathbf{A}_{2}+\ldots \leftrightharpoons n_{3} \mathbf{A}_{3}+\nu_{4} \mathbf{A}_{4}+\ldots
$$

em que $\mathbf{A}_{\mathrm{i}}$ são as espécies químicas envolvidas e $\nu_{\mathrm{i}}$ os coeficientes estequiométricos, positivos para os produtos e negativos para os reagentes. A condição de equilíbrio é traduzida pela variação de energia de Gibbs ser nula, isto é,

$$
\Delta \mathrm{G}=0 \quad(\mathrm{~T}, \mathrm{p}, \text { constantes })
$$

que se pode transformar em

$$
\Sigma \nu_{\mathrm{i}} \mu_{\mathrm{i}}=0
$$

em que $\mu_{\mathrm{i}}$ é o potencial químico do constituinte $i$. O problema reside agora no estabelecimento de $\mu_{\mathrm{i}}$. Dada a natureza diferencial da termodinâmica, $\mu_{\mathrm{i}}$ é conhecido a menos de uma constante. Assim, $\mu_{\mathrm{i}}$ é uma função de $\mathrm{T}$ e p que se pode calcular a partir da relação termodinâmica

$$
\left(\frac{\partial \mu_{\mathrm{i}}}{\partial \mathrm{p}}\right)_{\mathrm{T}}=\mathrm{V}_{\mathrm{i}}
$$

em que $V_{i}$ é o volume parcial molar do constituinte $\mathrm{i}$ na mistura. Conforme se trate duma mistura gasosa ou líquida, assim se processa a integração da equação (4). Vejamos o caso mais simples duma mistura gasosa perfeita. Neste caso, $\mathbf{V}_{\mathbf{i}}=\mathbf{V}_{\mathrm{i}}^{\mathbf{o}}$, isto é, o volume parcial molar reduz-se ao volume molar do gás puro, pelo que podemos integrar (4) entre um estado de referência $p_{i}^{\circ}$ e $\mathrm{p}_{\mathrm{i}}$, pressões parciais de $\mathrm{i}$ num estado padrão e no estado real, a que correspondem os potenciais químicos de i puro $\mu_{\mathrm{i}}^{\circ}(\mathrm{T})$ e de i na mistura $\mu_{\mathrm{i}}(\mathrm{T}, \mathrm{p})$

$$
\int_{\mu_{\mathrm{i}}(\mathrm{T})}^{\mu_{\mathrm{i}}(\mathrm{T}, \mathrm{p})} \mathrm{d} \mu_{\mathrm{i}}=\int_{\mathrm{p}_{\mathrm{i}}}^{\mathrm{p}_{\mathrm{i}}} \mathrm{V}_{\mathrm{i}}^{\mathrm{o}} \mathrm{d} \mathrm{p}
$$

pelo que, se utilizarmos a equação de estado dos gases perfeitos

$$
\mu_{\mathrm{i}}(\mathrm{T}, \mathrm{p})-\mu_{\mathrm{i}}^{\mathrm{o}}(\mathrm{T})=\mathrm{RT} \int_{\mathrm{p}_{\mathrm{i}}^{\mathrm{o}}}^{\mathrm{p}_{\mathrm{i}}} \frac{\mathrm{dp}}{\mathrm{p}}=\mathrm{RT} \ln \left(\frac{\mathrm{p}_{\mathrm{i}}}{\mathrm{p}_{\mathrm{i}}^{\mathrm{o}}}\right)
$$

A equação (6) pode agora ser introduzida na equação (3) obtendo-se

$$
\Sigma \nu_{\mathrm{i}} \mu_{\mathrm{i}}^{\mathrm{o}}+\mathrm{RT} \ln \prod_{\mathrm{i}}\left(\frac{\mathrm{p}_{\mathrm{i}}}{\mathrm{p}_{\mathrm{i}}^{o}}\right)^{\nu_{\mathrm{i}}}=0
$$

O primeiro termo desta equação é $\Delta \mathrm{G}^{\circ}$, a variação de energia de Gibbs na reacção quando todos os reagentes e produtos estão num estado padrão e o segundo termo define a constante de equilíbrio, isto é,

$$
\begin{aligned}
& \Delta \mathrm{G}^{\circ}=-\mathrm{RT} \ln \mathrm{K}_{\mathrm{p}} \\
& \mathrm{K}_{\mathrm{p}}=\prod_{\mathrm{i}}\left(\frac{\mathrm{p}_{\mathrm{i}}}{\mathrm{p}_{\mathrm{i}}^{\circ}}\right)^{\nu_{\mathrm{i}}}=\mathrm{e}^{-\Delta \mathrm{G}^{\circ} / \mathrm{RT}}
\end{aligned}
$$

A equação (8) mostra completamente a adimensionalidade da constante de equilíbrio, pois a razão $\mathrm{p}_{\mathrm{i}} / \mathrm{p}_{\mathrm{i}}^{\circ}$ é adimensional. $\mathrm{O}$ estado padrão dos gases escolhe-se para $\mathrm{p}_{\mathrm{i}}^{\circ}=1$ atm pelo que $\left(\mathrm{p}_{\mathrm{i}} / 1\right.$ atm) é adimensional, o que exige, como é lógico que $\mathrm{p}_{\mathrm{i}}$ venha sempre expresso em atmosferas.

Conclusão 1: A constante de equilíbrio duma reacção em fase gasosa é adimensional e deve ser introduzida como tal.

A generalização a qualquer reacção em fase líquida é imediata. De facto, retomando (4) e utilizando o mesmo processo

a) Departamento de Química, Faculdade de Ciências da Universidade de Lisboa, 1294 Lisboa Codex. 


$$
\int_{\mu_{\mathrm{i}}^{\mathrm{o}}(\mathrm{T}, \mathrm{p})}^{\mu_{\mathrm{i}}(\mathrm{T}, \mathrm{p}, \mathrm{x})} \int_{\substack{\text { componente puro, à mesma } \\ \mathrm{T} \text { e p da mistura líquida }}}^{\text {componente i na mistura }} \mathrm{V}_{\mathrm{i}} \mathrm{dP}
$$

Pode-se demonstrar $[3,4]$ que o integral do lado direito da equação (9) vale RT In $a_{i}$, em que $a_{i}$ é a actividade do constituinte i na solução, isto é,

$$
\mu_{\mathrm{i}}\left(\mathrm{T}, \mathrm{p}, \mathrm{x}_{\mathrm{i}}\right)-\mu_{\mathrm{i}}^{\mathrm{o}}(\mathrm{T}, \mathrm{p})=\mathrm{RT} \ln \mathrm{a}_{\mathrm{i}}
$$

A actividade é adimensional (quociente de duas fugacidades) pelo que se pode obter para a constante de equilíbrio:

$$
\begin{aligned}
& \Delta \mathrm{G}^{\circ}=\Sigma \nu_{\mathrm{i}} \mu_{\mathrm{i}}^{\circ}(\mathrm{T}, \mathrm{p})=-\mathrm{RT} \ln \mathrm{K}_{\mathrm{a}} \\
& \mathrm{K}_{\mathrm{a}}=\prod_{\mathrm{i}} \mathrm{a}_{\mathrm{i}}^{\nu_{\mathrm{i}}}=\mathrm{e}^{-\Delta \mathrm{G}^{\circ} / \mathrm{RT}}
\end{aligned}
$$

A constante de equilíbrio $\mathrm{K}_{\mathrm{a}}$ é a constante termodinâmica do equilíbrio em fase líquida e o índice $a$ designa a sua definição com base na actividade. Logo $\mathrm{K}_{\mathrm{a}}$ é adimensional.

Conclusão 2: A constante de equilíbrio duma reacção em fase líquida é adimensional. A $1 .^{\mathrm{a}}$ igualdade da equação (11) não deve ser tomada como definição de $\mathrm{K}_{\mathrm{a}}$ mas uma consequência da exponencial $\mathrm{e}^{-\Delta \mathrm{G} / \mathrm{RT}}$, que é necessariamente adimensional.

Vejamos agora em mais detalhe a aplicação da equação (11) a reacções em soluções electrolíticas, nomeadamente aos equilíbrios iónicos em soluções aquosas.

$\mathrm{O}$ valor de $\mathrm{K}_{\mathrm{a}}$ calcula-se a partir dos dados de energia de Gibbs padrão de formação dos iões em solução aquosa. O primeiro problema que surge é a escolha do estado padrão. Quando a solução é bastante diluída, isto é, quando um dos componentes está presente em muito maior quantidade (solvente), torna-se conveniente tratar este duma forma diferente. Assim considera-se para o solvente que o estado padrão é o líquido puro à mesma temperatura $\mathrm{T}$ e pressão $\mathrm{p}$ da solução, isto é

$$
\mu_{\mathrm{sol}}^{\mathrm{o}}=\mu_{\mathrm{sol}}^{*}(\mathrm{~T}, \mathrm{p})
$$

em que o asterístico designa puro. Então, a equação (10) escreve-se

$$
\mu_{\text {sol }}\left(\mathrm{T}, \mathrm{p}, \mathrm{x}_{\text {sol }}\right)=\mu_{\text {sol }}^{*}(\mathrm{~T}, \mathrm{p})+\mathrm{RT} \ln \mathrm{a}_{\text {sol }}
$$

A actividade $a_{i}$ de qualquer constituinte pode escrever-se na forma

$$
\mathrm{a}_{\mathrm{i}}=\gamma_{\mathrm{i}} \mathrm{x}_{\mathrm{i}}
$$

em que $\gamma_{\mathrm{i}}$ é o coeficiente de actividade da espécie i e $\mathrm{x}_{\mathrm{i}}$ a sua fracção molar. Designando por $\mathrm{m}_{\mathrm{i}}$ a molalidade da espécie i (moles/Kg de solvente), temos que

$$
\mathrm{m}_{\mathrm{i}}=\frac{\mathrm{n}_{\mathrm{i}}}{\mathrm{m}_{\text {solv }}}=\frac{\mathrm{n}_{\mathrm{i}}}{\mathrm{n}_{\text {solv }} \times \mathrm{M}_{\text {solv }}}=\frac{\mathrm{x}_{\mathrm{i}}}{\mathrm{x}_{\text {solv }} \mathrm{M}_{\text {solv }}}
$$

com $\mathrm{m}_{\text {solv }}$ expresso em $\mathrm{Kg}$ e $\mathbf{M}_{\text {solv }}$ expresso em $\mathrm{Kg}$ $\mathrm{mol}^{-1}$.
Para cada um dos solutos o seu estado padrão corresponde à sua diluição infinita no solvente, isto é,

$$
\lim _{\mathbf{x}_{\text {solv }} \rightarrow 1} \gamma_{i}=1
$$

o que transforma o estado padrão num estado fictício para o soluto onde ele não existe na solução, isto é

$$
\mu_{\mathrm{i}}^{\mathrm{o}}=\lim _{\mathrm{x}_{\text {solv }}-1}\left(\mu_{\mathrm{i}}-\mathrm{RT} \ln \mathrm{x}_{\mathrm{i}}\right)=\left(\mu_{\mathrm{i}}-\mathrm{RT} \ln \mathrm{x}_{\mathrm{i}}\right)^{\infty}
$$

Atendendo aos estados padrão podemos agora desenvolver as expressões dos potenciais químicos. Usando a equação (10) e (15), introduzindo $\mathrm{m}_{\mathrm{o}}$, molalidade padrão $\left(\mathrm{m}^{\circ} \equiv 1 \mathrm{~mol} / \mathrm{Kg}\right)$, vem

$$
\mu_{\mathrm{i}}=\mu_{\mathrm{i}}^{\mathrm{o}}+\mathrm{RT} \ln \left(\mathrm{M}_{\text {solv }} \mathrm{m}^{\mathrm{o}}\right)+\mathrm{RT} \ln \left(\mathrm{x}_{\text {solv }} \gamma_{\mathrm{i}} \frac{\mathrm{m}_{\mathrm{i}}}{\mathrm{m}_{\mathrm{o}}}\right)
$$

e definindo

$$
\begin{aligned}
& \mu_{\mathrm{m}, \mathrm{i}}^{\mathrm{o}} \equiv \mu_{\mathrm{i}}^{\mathrm{o}}+\mathrm{RT} \ln \left(\mathrm{M}_{\text {solv }} \mathrm{m}^{\mathrm{o}}\right) \\
& \gamma_{\mathrm{m}, \mathrm{i}} \equiv \mathrm{x}_{\mathrm{solv}} \gamma_{\mathrm{i}}
\end{aligned}
$$

vem

$$
\mu_{\mathrm{i}}=\mu_{\mathrm{m}, \mathrm{i}}^{\mathrm{o}}+\mathrm{RT} \ln \left(\gamma_{\mathrm{m}, \mathrm{i}} \frac{\mathrm{m}_{\mathrm{i}}}{\mathrm{m}^{\sigma}}\right)
$$

com

$$
\lim _{\substack{\mathrm{solv} \rightarrow 1 \\ \gamma_{\mathrm{m}, \mathrm{i}}}}=1
$$

que define o potencial químico dum soluto i em função do seu potencial químico no estado padrão caracterizado por $\mu_{\mathrm{m}, \mathrm{i}}^{\circ}$ e com uma dependência de $\mathrm{m}_{\mathrm{j}} / \mathrm{m}_{\mathrm{o}}$ análoga à que a equação (10) dá para a dependência de $\mathrm{x}_{\mathrm{i}}$. Nestas condições diz-se que se definem coeficientes de actividade em escala de molalidade. do-se

Para o solvente, podemos particularizar (13) obten-

$$
\mu_{\text {solv }}=\mu_{\text {solv }}^{\circ}+\text { RT } \ln \gamma_{\text {solv }} x_{\text {solv }}
$$

com

$$
\lim _{x_{\text {solv }}-1} \gamma_{\text {solv }}=1
$$

Convém notar que para a água, por exemplo, $\mathrm{M}_{\mathrm{A}} \mathrm{m}^{\circ}=0,018 \mathrm{Kg} \mathrm{mol}^{-1} \times 1 \mathrm{~mol} \mathrm{Kg}=0,018 \mathrm{e} \mathrm{x}_{\text {solv }}$ vem dado por

$$
\mathrm{x}_{\text {solv }}=\frac{\mathrm{n}_{\text {solv }}}{\mathrm{n}_{\text {solv }}+\mathrm{n}_{\mathrm{i}}}=\frac{1}{1+\frac{\mathrm{n}_{\mathrm{i}}}{\mathrm{n}_{\text {solv }}}}=\frac{1}{1+\mathrm{m}_{\mathrm{i}} \mathrm{M}_{\text {solv }}}
$$

e que para $\mathrm{m}_{\mathrm{i}}=1 \mathrm{~mol} / \mathrm{Kg}$, por exemplo $\mathrm{x}_{\text {solv }}=0,982 \sim 1$.

Após estas considerações podemos voltar ao problema do equilíbrio químico em soluções aquosas de electrólitos. Retomemos a equação (11) e as considerações anteriores. Podemos então escrever para a actividade da espécie i na escala de molalidades 


$$
a_{m, i}=\gamma_{m, i} \frac{m_{i}}{m^{\sigma}}
$$

em que a molalidade standard é $\mathrm{m}^{\circ}=1 \mathrm{~mol} / \mathrm{Kg}$, e para o solvente

$$
\mathrm{a}_{\text {solv }}=\gamma_{\text {solv }} \mathrm{x}_{\text {solv }}
$$

O valor de $\Delta G^{\circ}$ vem então

$$
\begin{aligned}
& \Delta \mathrm{G}^{\mathrm{o}}=\Sigma \nu_{\mathrm{i}} \mu_{\mathrm{i}}^{\mathrm{o}}=\nu_{\text {solv }} \mu_{\text {solv }}^{\mathrm{o}}+\sum_{\text {solutos }}^{\sum} \nu_{\mathrm{i}} \mu_{\mathrm{i}}^{\mathrm{o}} \\
& =\nu_{\text {solv }} \mu_{\text {solv }}^{\mathrm{o}}+\sum_{\text {solutos }}^{\sum} \nu_{\mathrm{i}} \mu_{\mathrm{m}, \mathrm{i}}^{\mathrm{o}}-\sum_{\text {solutos }} \nu_{\mathrm{i}} \mathrm{RT} \ln \left(\mathrm{M}_{\text {solv }} \mathrm{m}^{\mathrm{o}}\right) \\
& =\Delta \mathrm{G}_{\mathrm{m}}^{\mathrm{o}}-\mathrm{RT} \sum_{\text {solutos }}^{\Sigma} \nu_{\mathrm{i}} \ln \left(\mathrm{M}_{\text {solv }} \mathrm{m}^{\mathrm{o}}\right) \\
& \Delta \mathrm{G}^{\mathrm{o}}=\Delta \mathrm{G}_{\mathrm{m}}^{\mathrm{o}}-\mathrm{RT} \ln \left(\mathrm{M}_{\text {solv }} \mathrm{m}^{\mathrm{o}}\right) \sum_{\text {solutos }}^{\sum} \nu_{\mathrm{i}}
\end{aligned}
$$

em que $\Delta G_{m}^{o}$ é a variação de energia de Gibbs padrão, mas para o estado padrão na escala molalidades (estado fictício para os solutos). As tabelas termodinâmicas estão normalmente calculadas para nos dar o valor de $\Delta \mathrm{G}_{\mathrm{m}}^{\mathrm{o}}$, correspondente a uma actividade unitária de cada soluto (ou ião), uma vez que $\Delta G^{\circ}$ depende, através do $2 .^{\circ}$ termo do número de solutos (ou iơes) presentes e dos seus coeficientes estequiométricos. $25^{\circ} \mathrm{C}$

Assim, por exemplo, para uma solução aquosa a

$$
\Delta \mathrm{G}^{\circ}=\Delta \mathrm{G}_{\mathrm{m}}^{\circ}+\left(\sum_{\text {solutos }}^{\sum} \nu_{\mathrm{i}}\right)(9959 \mathrm{~J})
$$

A partir de $\Delta G_{m}^{o}$ podemos definir uma constante adimensional $\mathrm{K}_{\mathrm{m}}$, definida por

$$
\begin{aligned}
& \mathrm{K}_{\mathrm{m}}=\mathrm{e}^{-\Delta \mathrm{G}_{\mathrm{m}}^{\circ} / \mathrm{RT}}= \\
& =\left(\gamma_{\text {solv }} \mathrm{x}_{\text {solv }}\right)^{\nu_{\text {solv }}} \prod_{\text {solutos }}\left(\gamma_{\mathrm{m}, \mathrm{i}} \frac{\mathrm{m}_{\mathrm{i}}}{\mathrm{m}_{\mathrm{o}}}\right)^{\nu_{\mathrm{i}}}
\end{aligned}
$$

Vejamos a aplicação da equação ao equilíbrio da autoionização da água

$$
2 \mathrm{H}_{2} \mathrm{O} \leftrightharpoons \mathrm{H}_{3} \mathrm{O}^{+}+\mathrm{OH}^{-}
$$

Podemos escrever, usando o tradicional w para designar a água

$$
\begin{aligned}
\mathbf{K}_{\mathrm{m}}^{\mathrm{w}} & =\frac{\mathrm{a}_{\mathrm{H}_{3} \mathrm{O}^{+}} \times \mathrm{a}_{\mathrm{OH}^{-}}}{\mathrm{a}_{\mathrm{H}_{2} \mathrm{O}}^{2}} \\
\mathbf{K}_{\mathrm{m}}^{\mathrm{w}} & =\frac{\gamma_{\mathrm{H}_{3} \mathrm{O}^{+}} \frac{\mathrm{m}_{\mathrm{H}_{3} \mathrm{O}^{*}}^{\mathrm{m}^{\circ}} \times \gamma_{\mathrm{OH}^{*}} \cdot \frac{\mathrm{m}_{\mathrm{OH}^{-}}}{\mathrm{m}^{\circ}}}{\left(\gamma_{\mathrm{H}_{2} \mathrm{O}} \times_{\mathrm{H}_{2} \mathrm{O}}\right)^{2}}}{}
\end{aligned}
$$

Dum modo geral, e para soluções diluídas de electrólitos, que é o caso mais frequente, $\gamma_{\mathrm{H}_{2} \mathrm{O}} \sim 1$ e $\mathrm{x}_{\mathrm{H}_{2} \mathrm{O}} \sim 1$. Por outro lado, substitui-se $\gamma_{\mathrm{H}_{3} \mathrm{O} \cdot} \cdot \gamma_{\mathrm{OH}^{-}}$ por $\gamma_{ \pm}^{2}$, coeficiente de actividade médio, na escala de molalidades.
$\mathrm{O}$ valor de $\mathrm{K}_{\mathrm{m}}^{\mathrm{w}}$ pode calcular-se a partir dos dados tabelados de $\Delta \mathrm{G}_{\mathrm{f}}^{\mathrm{m}}$ dos diferentes iões. Assim, e considerando como referências que $\Delta \mathrm{G}_{\mathrm{f}}^{\mathrm{o}}\left(\mathrm{H}^{+}, \mathrm{aq}\right)=0$ e para a actividade unitária de todos os solutos como estado padrão, temos $\Delta \mathrm{G}_{\mathrm{f}}^{\mathrm{o}}\left(\mathrm{H}_{3} \mathrm{O}^{+}, \mathrm{aq}\right)=-237,19 \mathrm{~kJ} \mathrm{~mol}^{-1} \mathrm{e}$ $\Delta \mathrm{G}_{\mathrm{f}}^{\mathrm{o}}\left(\mathrm{OH}^{-}, \mathrm{aq}\right)=-157,27 \mathrm{~kJ} \mathrm{~mol}^{-1}$ e $\Delta \mathrm{G}_{\mathrm{f}}^{\mathrm{o}}\left(\mathrm{H}_{2} \mathrm{O}\right.$, liq) $=-273,19 \mathrm{~kJ} \mathrm{~mol}^{-1}$ (igual ao do $\mathrm{H}_{3} \mathrm{O}^{+}$, pois a do $\mathrm{H}^{+}$é considerada nula). Então

$$
\begin{aligned}
& \Delta \mathrm{G}_{\mathrm{m}}^{\circ}=\Delta \mathrm{G}_{\mathrm{f}}^{\circ}\left(\mathrm{H}_{3} \mathrm{O}^{+}, \mathrm{aq}\right)+ \\
& +\Delta \mathrm{G}_{\mathrm{f}}^{\circ}\left(\mathrm{OH}^{-}, \mathrm{aq}\right)-2 \times \Delta \mathrm{G}_{\mathrm{f}}^{\circ}\left(\mathrm{H}_{2} \mathrm{O}, \mathrm{liq}\right) \\
& =-273,19-157,27+2 \times 237,19=79,92 \mathrm{~kJ}
\end{aligned}
$$

$\mathrm{e}$

$$
\mathrm{K}_{\mathrm{m}}^{\mathrm{w}}\left(25^{\circ} \mathrm{C}\right)=\mathrm{e}-\frac{79920}{8,314 \times 298}=9,86 \times 10^{-15} \sim 10^{-14}
$$

Este é o valor que a experiência nos dá para $\mathrm{K}_{\mathrm{m}}^{\mathrm{w}}$. De notar no entanto que é o valor

$$
\gamma_{ \pm}^{2} \frac{\mathrm{m}_{\mathrm{H}_{3} \mathrm{O}^{+}} \mathrm{m}_{\mathrm{OH}^{-}}}{\left(\mathrm{m}^{\circ}\right)^{2}}=10^{-14}
$$

que se verifica e não, como muitas vezes se vê escrito

$$
\left[\mathrm{OH}^{-}\right]\left[\mathrm{H}_{3} \mathrm{O}^{+}\right]=10^{-14} \mathrm{~mol}^{2} \mathrm{l}^{-2}
$$

com $\left[\mathrm{OH}^{-}\right]$e $\left[\mathrm{H}_{3} \mathrm{O}^{+}\right]$as concentrações molares de cada ião expressas em mol $^{-1}$ (ou molaridade).

É evidente que, para soluções muito diluídas, com $\left.\mathrm{m}_{\mathrm{i}}<10^{-3}\right), \gamma_{ \pm} \sim 1 \mathrm{e} \mathrm{m}_{\mathrm{H}_{3} \mathrm{O}^{+}} \sim\left[\mathrm{H}_{3} \mathrm{O}^{+}\right], \mathrm{m}_{\mathrm{OH}^{-}} \sim\left[\mathrm{OH}^{-}\right] \mathrm{e}$ $\mathrm{m}^{\mathrm{o}} \sim \mathrm{C}^{\circ} \equiv 1 \mathrm{~mol} \mathrm{l}^{-1} \mathrm{e}$ virá

$$
\frac{\left[\mathrm{H}_{3} \mathrm{O}^{+}\right]\left[\mathrm{OH}^{-}\right]}{\left(\mathrm{C}^{0}\right)^{2}} \simeq 10^{-14}
$$

ou $\left[\mathrm{H}_{3} \mathrm{O}^{+}\right]=\left[\mathrm{OH}^{-}\right]=10^{-7} \mathrm{~mol} 1 \times 1$, e calculando $\gamma_{ \pm}$ pela fórmula de Davies obtém-se $\gamma_{ \pm}=0,9996$ para a água pura a $25^{\circ} \mathrm{C}$, o que é 1 .

As relações anteriores servem para demonstrar que, de facto, a constante de equilibrio duma reacção química, em fase gasosa ou liquida é sempre adimensional e que deve ser ensinada e introduzida a nível do ensino secundário como tal. A análise dimensional pode ser útil na sua introdução.

A generalização da constante de equilíbrio a toda a química de solução é imediata, desde constantes de acidez e basicidade a produtos de solubilidade.

\section{BIBLIOGRAFIA}

[1] - C.D. MiCKey, J. Chem. Ed., 57, 801 (1980).

[2] - P.G. WRIGHT, Education in Chemistry, 2, 14 (1965).

[3] - J.M. SMITH, H.C. VAN Ness, Introduction to Chemical Engineering Thermodynamics, ed. Mc Graw-Hill (1975).

[4] - I.N. Levine, Physical Chemistry, ed. Mc Graw-Hill (1979).

\section{Então}

$$
\mathrm{K}_{\mathrm{m}}^{\mathrm{w}}=\gamma_{ \pm}^{2} \frac{\mathrm{m}_{\mathrm{H}_{3} \mathrm{O}^{\cdot} \cdot \mathrm{m}_{\mathrm{OH}^{-}}}}{\left(\mathrm{m}^{\circ}\right)^{2}} \quad \text { (soluções diluídas) }
$$

